\title{
Using Trees to Remediate Groundwaters Contaminated with Chlorinated Hydrocarbons
}

\author{
US DOE, Grant No. DE-FGO7-96-ER20256 \\ Stuart E. Strand", Milton P. Gordon', Lee A. Newman \\ "College of Forest Resources, 'Dept. Biochemistry \\ University of Washington, Seattle WA 98195
}

Annual Progress Report, 1996-97

Metabolism of Chlorinated Hydrocarbons

Laboratory and field tests with poplar in tissue culture, bioreactors, and field sites have shown that, unlike bacteria, these plants are able to carry out complete degradation of fully chlorinated alkanes and alkenes to carbon dioxide and chloride. Carbon dioxide was produced as a product of the degradation of trichloroethylene (TCE), carbon tetrachloride (CT), and perchloroethylene (PCE) when axenic tissue cultures of poplar cells were exposed to radiolabelled compounds. The apparent degradation of PCE and $\mathrm{CT}$, fully chlorinated hydrocarbons, in these aerobic plants is remarkable when contrasted to the lack of comparable aerobic degradation by bacteria. Oxidized metabolites, such as trichloroethanol, and di- and trichloroacetic acid, were detected in cell cultures exposed to TCE, suggesting the involvement of cytochrome P450s or other monooxygenase activities. Mass balance experiments with small poplar plants in laboratory reactors showed that significant TCE and CT was volatilized from the leaves, while a similar fraction of radiolabeled carbon from these chlorinated solvents was retained in the plant tissue.

In late 1996 we discovered that large poplar growing in full sun took up trichloroethylene (TCE) and carbon tetrachloride (CT) without significant solvent transpiration. Pilot scale experiments in the field were carried out using cells containing approximately $12 \mathrm{~m}^{3}$ soil with an underlying sand layer through which an artificially contaminated groundwater flowed. Hybrid poplar were planted in these cells (15 trees per cell). TCE or CT was added to the influent of the cells at concentrations ranging from 1 to $100 \mathrm{mg} / \mathrm{L}$, but generally averaging $15 \mathrm{mg} / \mathrm{L}$. Control cells consisted of poplar planted cells with no solvent exposure and cells with TCE or CT exposure but no vegetation. Mass removal of TCE and CT from the groundwater in these cells exceeded 95\% . Laboratory tests of soil from the cells showed that there was no enhancement of TCE and CT degradation in the rhizosphere soils. Transpiration of TCE and CT by the hybrid poplar on the test site was measured by two methods: longpath open FTIR and by bagging leaves and trapping solvent vapors. By both methods air emissions of the chlorinated solvents were less than $6 \%$ of the total removal. $94 \%$ 
of the total chloride introduced to the site, mostly as TCE and CT, was recovered, primarily as chloride ion in the soils, suggesting that significant dechlorination occurred. Although some chlorinated metabolites (e.g., chloroacetic acids and trichloroethanol) were found in plant tissues, there was no significant increase in total organic halides in the tissue of trees exposed to solvent compared to unexposed controls. We conclude that poplar have the potential for destructive removal of TCE and CT without harmful air emissions or accumulation of a hazardous solid waste.

Ongoing experiments include tests of the degradative abilities of willow, various poplar, and black locust, Willow, especially, have fixed significantly large amounts radiolabelled carbon from TCE and chlorinated benzene in laboratory .

Genetic Experiments

Tobacco and poplar have been transformed with the genes for mammalian cytochrome P450 2E1, cytochrome b5 and the cytochrome P450 oxidoreductase behind Mac promoters in a Agrobacterium vector. This construct was created because attempts to introduce the cytochrome P450 alone into poplar failed to achieve a protein product, presumably due to instability in the absence of the other members of the normal mammalian P450 complex. Both transformants have been obtained and, when shooted, they will be tested for the presence of the genes, mRNAs, proteins and activities. 